\section{El voluntariado como espacio formativo en competencias interculturales. Un aporte para la integralidad de la formación universitaria}

\author{
Gracia María Clérico \\ graciamariaclerico@gmail.com \\ (iD) orcid.org/0000-0001-5823-326X \\ Patricia Ingüi \\ patringui@gmail.com \\ (iD) orcid.org/0000-0003-3353-2575 \\ Universidad Nacional del Litoral, \\ Argentina.
}

RECEPCIÓN: 24/06/19

ACEPTACIÓN FINAL: 15/10/19

\section{Resumen}

Se presenta una intervención en Santa $\mathrm{Fe}$, en el marco de una experiencia de extensión de la Universidad Nacional del Litoral, focalizada en la inclusión socioeducativa y cultural de adolescentes de poblaciones socialmente vulnerables que se lleva adelante desde 2009 en forma conjunta con la Asociación Cultural para el Desarrollo Integral, a la que recientemente se incorporaron otras dos organizaciones no gubernamentales: Asociación de Artistas Plásticos Santafesinos y Asociación Civil "Espacios Ekekos". La trama tejida en este recorrido ofrece interesantes modalidades para contribuir a la integralidad de la formación universitaria. Esta brinda una contribución significativa para la formación en competencias interculturales de los estudiantes que participan como voluntarios.

Palabras clave: voluntariado universitario, adolescencia, educación en competencias interculturales, integralidad de la formación universitaria, inclusión socioeducativa y cultural.
Volunteering as a training space in intercultural competences. A contribution for the integrality of university education

\section{Abstract}

An intervention in Santa $\mathrm{Fe}$ is presented, within the framework of an extension experience focused on the socio-educational and cultural inclusion of adolescents from socially vulnerable populations, which has been carried out since 2009 jointly with the Cultural Association for Integral Development. Recently were incorporated another two non-governmental organizations: Association of Plastic Artists Santafesinos and Civil Association "Ekekos Spaces". The net woven in this route offers possible modalities to contribute to the integrality of the university education. She provides a significant contribution to the intercultural competence training of university students who participate as volunteers.

Keywords: university volunteering, adolescence, education in intercultural competences, integrality of university education, socio-educational and cultural inclusion.
Cultura(s) en clave de extensión universitaria
O voluntariado como espaço de formação em competências interculturais. Uma contribuição para a integralidade do ensino universitário

\section{Resumo}

Apresenta-se uma intervenção em Santa Fé, no contexto de uma experiência de extensão da Universidade Nacional del Litoral, focada na inclusão socioeducativa e cultural de adolescentes de populações socialmente vulneráveis, e que vem sendo realizada em parceria com a Associação Cultural para o Desenvolvimento Integral, desde 2009, tendo incorporado recentemente outras duas organizações não governamentais: Associação de Artistas Plásticos Santafesinos e Associação Civil Espaços EKEKOS. A trama tecida neste percurso oferece modalidades interessantes para contribuir para a integralidade da formação universitária. Ela fornece uma contribuição significativa para o treinamento em competências interculturais dos alunos que participam como voluntários.

Palavras-chave: voluntariado universitário, adolescência, educação em competências interculturais, integralidade do ensino universitário, inclusão socioeducativa e cultural.

Para citación de este artículo: Clérico, G.; Bonelli, M y Ingüi, P. (2019). El voluntariado como espacio formativo en competencias interculturales. Un aporte para la integralidad de la formación universitaria. +E: Revista de Extensión Universitaria, 9(11), 110-129. doi: 10.14409/extension.v9i11. Jul-Dic.8717. 


\section{Introducción}

Presentamos este artículo ${ }^{1}$ con el propósito de compartir una lectura valorativa de una experiencia extensionista en curso llevada a cabo a lo largo de una década por un equipo interdisciplinario de la Universidad Nacional del Litoral (UNL) de Santa Fe, Argentina. La misma se desarrolla en dos líneas centrales vinculadas a la inclusión educativa: la cuestión referida al aprendizaje del oficio estudiantil y la educación artística. Los destinatarios de estas iniciativas son adolescentes y jóvenes de escuelas que atienden a poblaciones socialmente vulnerables en Santa Fe.

A lo largo del camino recorrido se fueron integrando a los equipos de extensión docentes y egresados de las Facultades de Humanidades y Ciencias, Bioquímicas y Ciencias Biológicas, Ciencias Económicas, Ciencias Jurídicas e Ingeniería Química de UNL. A su vez, los estudiantes que vienen participando como voluntarios pertenecen prácticamente a todas las facultades de dicha Universidad. En forma reciente, y en virtud de la articulación de estas acciones con las de una propuesta de internacionalización curricular, se implican también docentes de otras universidades: Universidad Autónoma de Entre Ríos (UADER), de Argentina, Universidad Federal de Mina Gerais (UFMG) y Universidad Federal dos Vales do Jequitinhonha e Mucuri (UFVJyM), de Brasil.

En este trabajo damos a conocer diversos aspectos acerca de cómo se originó y cómo implementamos la propuesta, así como su posible contribución para la integralidad de la formación universitaria de los estudiantes involucrados, especialmente relativa a la educación intercultural. Para ello compartimos una aproximación a la cuestión intercultural y comentamos información recogida en diversas instancias donde los actores describen y evalúan las actividades desplegadas. Los resultados obtenidos ofrecen elementos que permiten reconocer un triple impacto del voluntariado en relación con la educación en competencias interculturales: fortalece la empatía y la apertura hacia el otro, propicia el diálogo intercultural e incentiva la actitud crítica y reflexiva. De esta manera, se evidencia su valiosa contribución para la integralidad de la formación universitaria.

A través de este trayecto invitamos a pensar y repensar acerca del potencial educativo de las experiencias de voluntariado; específicamente, esperamos que sea una contribución para reflexionar sobre los alcances interculturales de las prácticas de extensión.

\section{Una mirada a la historia}

Esta intervención en territorio tuvo sus inicios en el año 2009, en el marco de una acción conjunta entre UNL y el Programa Padrinos Solidarios desarrollado por la Asociación Cultural para el Desarrollo Integral (ACDI), que consiste en un programa de padrinazgo a distancia en el que una persona (o un grupo de personas) que vive en otra ciudad ayuda económicamente a un niño/a o adolescente en situación de vulnerabilidad social a través de diversas actividades de educación y promoción social. Dicho programa permite facilitar el acceso a educación, atención de la salud, alimento y vestimenta, pero sin alterar las condiciones actuales de vida del niño o niña, para que pueda crecer en su entorno y en el respeto de su familia.

1) Se trata de un avance de la investigación CAl+D 2016: "La interculturalidad en la internacionalización de la educación superior: estudio de una experiencia entre universidades de Argentina y Brasil", aprobada por Resolución del Consejo Superior 48/17 de la Universidad Nacional del Litoral. 
El interés fundamental es colaborar con el crecimiento de los niños no solo a nivel material sino compartiendo las necesidades y ayudando a ampliar su horizonte de posibilidades a través de la educación. Los padrinazgos a distancia son coordinados en mayor medida por trabajadores sociales o docentes que siguen de cerca la realidad de los niños y conocen su situación, su familia y sus necesidades. Por eso esta ayuda puede ser más que material, ya que la pequeña cuota que aporta el padrino posibilita el trabajo de toda una red de personas involucradas en la educación de esos niños. ${ }^{2}$

Una de las instituciones destinatarias de ese Programa, la escuela secundaria Particular Incorporada № 2035 Santa Rosa de Lima, del barrio homónimo de la ciudad de Santa Fe, solicitó a los responsables del Programa Padrinos Solidarios la inclusión de propuestas de apoyo escolar para acompañar a los estudiantes con mayores dificultades en sus trayectorias escolares. Este requerimiento, derivado de los altos índices de fracaso que registraba esa población escolar, llevó a que ACDI convocara a docentes de UNL para colaborar en la formulación de una estrategia de abordaje.

Así nace esta propuesta extensionista, y como resultado de esa preocupación inicial se lleva a cabo la escritura conjunta entre las tres instituciones (escuela, ACDI y UNL) de un proyecto presentado a la convocatoria del Programa de Voluntariado Universitario de la Secretaría de Políticas Universitarias del Ministerio de Educación, Cultura, Ciencia y Tecnología de la Nación, el que desde entonces viene aprobando y sosteniendo esta iniciativa en sus sucesivas versiones.

Durante este trayecto se incorporaron nuevas instituciones educativas, algunas que forman parte del Programa Padrinos Solidarios de ACDI y otras que expresaron su interés por participar. ${ }^{3}$ Esta propuesta es acogida por las autoridades escolares puesto que en ella encuentran la posibilidad de un apoyo concreto a los desafíos que presenta la tarea educativa desarrollada en contextos de vulnerabilidad social.

Incluyendo el año en curso, han participado hasta el momento 12 escuelas de la ciudad de Santa Fe y su zona de influencia (fundamentalmente de nivel medio) que se caracterizan por atender a adolescentes y jóvenes en riesgo educativo.

Es preciso resaltar que, además de la demanda por parte de nuevas instituciones educativas, este crecimiento responde a un hecho imprevisto y sostenido en el tiempo pero que constituye cada año una novedad para el equipo extensionista: la gran cantidad de estudiantes que se inscribe en respuesta a las convocatorias anuales de voluntariado universitario de la Secretaría de Extensión de la UNL. Ellos participan en el marco del Régimen de Voluntariado que propone la UNL para tal fin, ${ }^{4}$ que incluye la firma de un acta acuerdo donde se establecen las condiciones para garantizar la prestación de su tarea. Para llevar adelante este trabajo, invitamos a estudiantes a participar en carácter de voluntarios para desempeñarse en el rol de tutores.

2) Véase: http://acdi.org.ar/padrinos-solidarios

3) Escuelas de nivel primario: Escuela Particular Incorporada $N^{\circ} 1225$ Ceferino Namuncurá; Escuela Particular Incorporada N 1132 Cristo Obrero; Escuela Particular Incorporada N 1224 Nuestra Señora de Itatí; Anexo Varadero Sarsotti de la Escuela N ${ }^{\circ} 1224$ Nuestra Señora de Itatí. Escuelas de nivel medio: EESPI No 2035 Santa Rosa de Lima; EESOPI N² 2025 Ceferino Namuncurá; EESPI N³138 Santa Lucía; EETP № 481 Esteban Echeverría; EESO № 488 San Agustín, de la localidad de San Agustín; EESOPI №3025 Cristo Obrero; EESPI № 3098 Juan Marcos; EESO N 496 Héroes del 2 de Abril, de la localidad de Desvío Arijón.

4) Véase: Régimen de Voluntariado Universitario, UNL: https://www.unl.edu.ar/extension/wp-content/uploads/sites/9/2018/11/1243_regimen_de_voluntariado.pdf 
Cada año se suma un grupo importante de voluntarios de diferentes carreras e incluso de otras instituciones educativas. En efecto, se incorporan como voluntarios sociales de ACDI estudiantes de institutos de formación docente ${ }^{5}$ (de nivel superior no universitario), quienes ofrecen su apoyo en campos del saber con los que aún no cuenta la Universidad y participan activamente en todas las acciones, en forma conjunta con los voluntarios de UNL.

De tal manera, este proyecto, que originalmente se tituló: "Tutorías de apoyo escolar para adolescentes en situación de pobreza de Santa Fe" y actualmente lleva el nombre "Enseñar y aprender a estudiar", fue ampliando sus alcances en estos diez años, comprometió a más de 250 voluntarios y benefició directamente a 12 escuelas y más de 1000 alumnos.

Para abordar las demandas surgidas en el territorio, en forma reciente fueron convocadas otras organizaciones no gubernamentales. Entonces, desde 2018 la Asociación de Artistas Plásticos Santafesinos, y desde 2019 la Asociación Civil "Espacio Ekekos", participan en la segunda línea: la educación artística de los alumnos de estas escuelas. Esta iniciativa asumió el formato de Proyectos de Interés Social (PEIS) ${ }^{6}$ aprobado por la Secretaría de Extensión de UNL. La incorporación de estas ONG permite el contacto directo de los jóvenes con artistas del medio, lo que enriquece su bagaje cultural.

La coordinación de estos proyectos fue siempre llevada adelante de modo colaborativo con ACDI; por lo tanto, la articulación entre ambas instituciones — UNL y ACDI— se sostuvo a lo largo del tiempo, lo cual se plasmó en el accionar conjunto tanto durante las instancias de formulación de los proyectos como en la ejecución de las sucesivas propuestas de intervención. Este vínculo se formalizó en convenios de mutua colaboración ratificados anualmente por sus autoridades.

Esta propuesta, como se dijo, tuvo sus inicios en 2009 y dio comienzo a un trabajo en red que durante esta década fue multiplicando los actores participantes. En este itinerario también se diversificaron las estrategias, lo que permitió abordar las demandas emergentes en el territorio como también responder a las sugerencias derivadas de las evaluaciones de cada período.

\section{Semblanzas de la experiencia extensionista}

Los dispositivos de intervención fueron variando conforme a los requerimientos de las escuelas y sobre la base de las sucesivas evaluaciones realizadas con los actores implicados. Comenzamos con la implementación de tutorías de apoyo escolar en horarios extraáulicos, luego incorporamos el acompañamiento al docente en el aula y, finalmente, talleres de creación artística.

La respuesta masiva a las convocatorias de voluntarios da cuenta del valor que asignan los estudiantes a prácticas de esta índole, en las que pueden poner en juego sus incipientes saberes profesionales. Al mismo tiempo, pone en evidencia la sensibilidad social de 
los jóvenes respecto de las problemáticas y necesidades de los habitantes de la ciudad en situaciones más vulnerables. De tal modo, contribuyen a cuestionar las características que, desde los prejuicios, muchos adultos atribuyen a las nuevas generaciones; generalmente rasgos de indiferencia, falta de compromiso o irresponsabilidad. Las actividades que desempeñan a lo largo del año incluyen la difusión de la propuesta en los espacios escolares, la implementación de tutorías extraescolares, el acompañamiento en tareas áulicas o bien en talleres artísticos (siempre en áreas disciplinares vinculadas a su formación profesional). Estas acciones se complementan con la participación en los cursos de capacitación y el monitoreo y evaluación. Cada uno desarrolla este voluntariado asociado a su propia carrera, poniendo sus conocimientos y competencias a disposición.

Actualmente, esta acción extensionista tiene dos facetas centrales: por un lado, se brindan tutorías a adolescentes en contextos escolares de vulnerabilidad social. A través de ellas se intenta atender las necesidades de un gran número de alumnos que muestra problemas de aprendizaje como, por ejemplo: lenta alfabetización, dificultades en la comprensión y en la producción oral y escrita, así como insuficientes habilidades para estudiar y, sobre todo, para concebirse como estudiantes.

Por otro lado, y en forma complementaria, se ofrecen propuestas de expresión artística para esos mismos adolescentes en talleres coordinados por estudiantes universitarios especializados (seleccionados según el sistema de Becas de Extensión de UNL), quienes trabajan junto a otros estudiantes voluntarios. Con esta propuesta se procura atender a la escasa oportunidad de acceso a propuestas artísticas y culturales que tiene esta población y ampliar su horizonte simbólico.

La participación de las asociaciones civiles vinculadas al campo artístico, la Asociación de Artistas Plásticos Santafesinos y el Espacio Civil Ekekos, permite enriquecer estas acciones con la colaboración de artistas de reconocida trayectoria.

Considerando que una nace en el seno de la otra, ambas líneas se encuentran ligadas por orientarse hacia un mismo destinatario -adolescentes y jóvenes-, concretarse en un mismo contexto socioeducativo de ejecución y mediante una misma coordinación responsable de su ejecución: UNL y ACDI.

Además de acompañar a los adolescentes y mejorar la calidad de sus aprendizajes, un objetivo de conjunto, central en esta propuesta de intervención, es contribuir con la formación académica, profesional y personal de los estudiantes universitarios y, al mismo tiempo, enriquecer la labor de los docentes de las escuelas destinatarias.

La atención a estos objetivos nos llevó a desarrollar como dispositivo el dictado anual de cursos de capacitación. Precisamente allí pudimos alojar las solicitudes de los estudiantes, quienes plantearon su necesidad de contar con un espacio de orientación para su labor en terreno. Es importante aclarar que muchos de ellos pertenecen a carreras universitarias no vinculadas con la formación docente, por lo que expresan inquietudes relativas a elementos pedagógicos y didácticos para elaborar conceptualmente algunos emergentes de las prácticas y entonces fortalecer su posicionamiento en las escuelas.

Compartimos las acciones de capacitación entre los integrantes de los equipos extensionistas de ambas líneas de trabajo, con el apoyo como asesores externos de colegas de 
las otras tres universidades: UFMG y UFVJyM (Brasil) y UADER (Argentina). ${ }^{7}$ Los contenidos van variando según los requerimientos de cada etapa, focalizándonos en la cuestión del desarrollo de habilidades ligadas al estudio y de la educación artística en contextos de vulnerabilidad social.

Asimismo, estos cursos ofrecen a los estudiantes la oportunidad de enriquecerse en el intercambio con sus pares mediante el reconocimiento de puntos en común y el fortalecimiento de los aportes con relación a su intervención y a su formación profesional.

De esa manera, intentamos contribuir a la profesionalización de sus prácticas, lo cual constituye uno de los rasgos del voluntariado actual que, superando la visión tradicional desde la cual bastaba la "buena voluntad", propende a ofrecer formación adecuada para desempeñar las tareas (Gaete Quezada, 2015).

De acuerdo también con sus inquietudes, cada año desarrollamos actividades destinadas a los docentes de las escuelas involucradas. ${ }^{8}$ Además de favorecer la coordinación entre las propuestas didácticas de los docentes y la labor del voluntario, esto nutre nuestra visión extensionista ya que nos permite una mirada más ajustada de las necesidades y los recursos de ese contexto. Sin dudas, la participación de docentes de las escuelas junto a los estudiantes universitarios en estas acciones de capacitación posibilita potenciar los alcances de esta intervención.

\section{Aproximándonos a la cuestión intercultural}

Inevitablemente, las fronteras culturales se desplazan y, por lo tanto, el ritmo de las transformaciones sociales aumenta. Como resultado, la diversidad cultural y el contacto intercultural constituyen hechos de la vida moderna y las competencias interculturales han empezado a ser una respuesta necesaria (UNESCO, 2017).

La cuestión intercultural entra en juego cuando existe encuentro entre personas y grupos pertenecientes a culturas diferentes.

Sin el interés de realizar una delimitación exhaustiva, para ayudar a esclarecer la noción de cultura que actualmente es reexaminada de manera crítica en las ciencias sociales, comenzamos retomando a Leite (2016), quien, a partir de las contribuciones fenomenológicas de Ales Bello (1998), sostiene:

"A cultura pode ser descrita como a actividade que manipula a realidade segundo um projeto e, nesse processo, contribui para a constituição dessa realidade de um determinado modo. Trata-se, portanto, de reconhecê-la como prolongamento de posicionamentos pessoais e coletivos de constituição do mundo, isto é, como realidade objetiva que nasce da atividade humana e permanece no tempo solicitando novas gerações". (p. 121)

7) Participan como capacitadores en los Cursos de capacitación, junto con Patricia Ingüi, Teresita Prat y Gracia María Clérico (UNL), los profesores Yuri Gaspar y Roberta Vasconcelos de la Universidad Federal dos Vales do Jequitinhonha e Mucuri (UFVJyM); Raquel Assis, Ana Lydia Santiago y Liberia Neves, de la Universidad Federal dos Universidad Federal de Mina Gerais (UFMG); y Mariana Bonelli de UADER.

8) Así, se vienen desarrollando con los docentes de las escuelas involucradas diversos talleres, cursos de extensión, foros interinstitucionales, conferencias y videoconferencias con expertos extranjeros. 
De tal manera, la cultura se constituye como la fuente de sentidos con que damos significados a los fenómenos de la vida cotidiana para poder interactuar socialmente, tal como lo indica Clifford Geertz (2000) al considerarla como la urdimbre de la trama de significaciones que el hombre va tejiendo. El análisis de la cultura es, entonces, una "ciencia interpretativa que busca significaciones" (p. 20). Y en tanto "se debe considerar el encuentro de culturas desde una perspectiva situacional que no oculte que se pone en juego lo social" (Cuche, 2002:84), podemos poner de relieve que:

"No hay verdadera discontinuidad entre las culturas, que están cada vez más en comunicación unas con otras, por lo menos al interior de un espacio social dado. Las culturas particulares no son totalmente extrañas entre sí, incluso cuando acentúan sus diferencias para afirmarse mejor y distinguirse unas de otras". (Amselle, 1990, citado en Cuche:83)

Resulta asimismo valioso para profundizar esta temática introducir la contribución de Pierre Bourdieu, quien, para referirse a la cultura en sentido antropológico acude, en su obra Le Sens Pratique, a la noción de habitus:

"Los sistemas de disposiciones duraderas y transmisibles, estructuras estructuradas predispuestas a funcionar como sistemas estructurantes, es decir, en tanto principios generadores y organizadores de prácticas y de representaciones que pueden ser objetivamente adaptadas a su objetivo y sin suponer una meta consciente de fines y de dominio expreso de las operaciones necesarias para alcanzarlas". (1980:88, citado en Cuche:102)

Sin que Bourdieu considere al habitus como un sistema rígido de disposiciones, que determinaría de manera mecánica las representaciones y las acciones, estima que

"a las diferentes posiciones en un espacio social dado le corresponden estilos de vida que son la expresión simbólica de las diferencias inscriptas objetivamente en las condiciones de existencia. (...) El habitus es también incorporación, en sentido propio, de memoria colectiva". (Cuche:102)

Esta profundización de la idea de cultura conduce a examinar la noción de identidad, tan "difícil de delimitar y definir por su carácter multidimensional y dinámico. Esto es lo que le confiere su complejidad, pero también le otorga flexibilidad" (130). Es preciso considerar además que "adoptar un enfoque puramente objetivo o puramente subjetivo para abordar la cuestión de la identidad es encerrarse en un callejón sin salida" (109).

La concepción dinámica de identidad propugnada por Barth (1969, citado en Cuche, 2002) como manifestación relacional permite superar la alternancia objetivismo/subjetivismo. La identidad es siempre una relación con el otro. Dicho de otro modo, identidad y alteridad tienen una parte en común y están en una relación dialéctica. Para Barth, hay que buscar aprehender el fenómeno identitario en el orden de las relaciones entre los grupos sociales. La diferencia identitaria no es consecuencia directa de la diferencia cultural (Cuche, 2002).

En los términos de Bourdieu (1980, citado en Cuche, 2002), el concepto de estrategia identitaria indica que el sujeto, como actor social, no carece de cierto margen de maniobra; la identidad se construye a través de las estrategias de los actores sociales. Si se quiere considerar que el otro no es nunca otro de manera absoluta, que hay algo de mismo en el otro, porque la humanidad es una, lo que hace que la cultura esté en el centro de las culturas 
o, según la expresión famosa, "lo universal está en el centro de lo particular", entonces es posible concebir con Bourdieu que: "Si dejamos de ser proyecciones más o menos complacientes, la etnología y la sociología llevan al descubrimiento de uno mismo en y por la objetivación de uno que exige el conocimiento del otro" (1985:79, citado en Cuche:149).

Así, "el investigador aprehende la dialéctica de lo mismo y del otro, de la identidad y de las diferencias, dicho de otro modo, de la Cultura y de las culturas, fundamento de la dinámica social” (149).

Para avanzar en la temática aquí abordada es necesario destacar que el crecimiento de la preocupación por la temática multicultural ${ }^{9}$ atraviesa diversos ámbitos disciplinarios y asume tres acentos continentales: anglosajón, europeo continental y latinoamericano (Dietz y Mateo Cortés, 2011). En este sentido, podemos destacar:

"En el contexto latinoamericano, las propuestas educativas para la diferencia se orientaron más hacia la interculturalidad que hacia el multiculturalismo. El prefijo 'inter' supone no un hecho dado, sino una realidad que se construye en la interrelación de las culturas, que requiere el diálogo y la interacción desde donde se construye la diferencia". (Soler Castillo, 2018:27)

En los documentos preparatorios para la reciente Conferencia Regional de Educación Superior (CRES, 2018) organizada por UNESCO-IESALC se precisan los alcances de esta posición en nuestro continente:

"En la actualidad, en América Latina, los usos más frecuentes de las ideas de interculturalidad se registran en los campos de Educación y Salud. En educación, en particular, la idea a menudo se utiliza con referencia a propuestas y programas de Educación Intercultural, los cuales además se distinguen de la idea de inclusión, porque reconocen y valoran la existencia de diferencias significativas entre diversos modos de producir conocimientos, modalidades de aprendizaje, valores y proyectos de futuro". (Mato, 2018:13)

Para entender las diferencias entre las distintas posturas, podemos recuperar la propuesta de Giménez Romero (2003) retomada por Dietz (2011) y Dietz y Mateos Cortés (2012), quienes indican que se requiere distinguir entre el plano de los hechos y el de las propuestas sociopolíticas y éticas para separar conceptualmente los discursos descriptivos o analíticos de la inter o multiculturalidad de los discursos propositivos o ideológicos.

Desde esta óptica, Dietz (2012), citado en Clérico (2014), establece una diferencia central entre el multiculturalismo y la postura intercultural: el reconocimiento de la diferencia en términos de respetar el principio de igualdad y diferencia cultural, de la perspectiva multicultural, y la posibilidad de una convivencia en la diversidad en la perspectiva intercultural. Esta se basa también en dichos principios, pero incluye la posibilidad de que se desarrolle una interacción positiva entre los diversos grupos culturales. Esta distinción queda plasmada a

9) Cabe señalar que la educación multicultural y la educación intercultural "no han nacido como construcciones de una teoría determinada ni se han traducido en un modelo teórico práctico específico de intervención educativa" (Muñoz Sedano, 1999:205). Devinieron, en cambio, del interés por responder al viejo problema de las relaciones entre los pueblos (Clérico, 2014). Esto muestra lo complejo que resultaría ofrecer una síntesis acabada de ambas posturas. Lo mismo ocurre con la amplitud de modelos y programas educativos que se despliegan en el campo de la educación multi e intercultural. Dar cuenta exhaustiva de ambas cuestiones excede los propósitos de este artículo. 
nivel fáctico en el acento puesto en el primer caso, en la diversidad como tal; en el segundo, en las interrelaciones entre las culturas. La comparación entre ambas posturas en cuanto a la cuestión del pluralismo diferencia su posición en el plano fáctico y normativo, tal como se reconoce en el cuadro siguiente:

Cuadro 1. Comparación de posición ante el pluralismo de enfoques multicultural e intercultural

\begin{tabular}{|c|c|c|}
\hline Plano / Enfoque & Multicultural & Intercultural \\
\hline $\begin{array}{l}\text { PLANO FÁCTICO } \\
\text { o de los hechos } \\
=\text { lo que es }\end{array}$ & $\begin{array}{l}\text { MULTICULTURALIDAD } \\
\text { Diversidad cultural, lingüística, religiosa. }\end{array}$ & $\begin{array}{l}\text { INTERCULTURALIDAD } \\
\text { Relaciones interétnicas, interlingüísticas, } \\
\text { interreligiosas. }\end{array}$ \\
\hline $\begin{array}{l}\text { PLANO NORMATIVO } \\
=\text { lo que debería ser }\end{array}$ & $\begin{array}{l}\text { MULTICULTURALISMO } \\
\text { Reconocimiento de la diferencia. } \\
\text { 1. Principio de igualdad. } \\
\text { 2. Principio de diferencia. }\end{array}$ & $\begin{array}{l}\text { INTERCULTURALISMO } \\
\text { Convivencia en la diversidad. } \\
\text { 1. Principio de igualdad. } \\
\text { 2. Principio de diferencia. } \\
\text { 3. Principio de interacción positiva. }\end{array}$ \\
\hline
\end{tabular}

Fuente: Dietz (2012:16).

Puesto que "apuesta al intercambio, la reciprocidad, la interacción entre culturas" (Malgesini y Giménez, 2000:128), el interculturalismo no busca tanto la diferenciación entre ellas como su interacción. Sin embargo, "ninguna cultura es considerada superior a otra y con derecho a dominarla, pero tampoco comparte con los relativistas que todas valgan por igual" (Sales Ciges y García López, 1997:20).

Así, la interculturalidad asume la diferencia no solo como algo necesario sino como algo virtuoso, ya que incluye comprensión y respeto entre las culturas (Schmelkes, 2003). En este sentido, la educación intercultural subraya la interrelación dinámica entre las mismas y los reacomodos a ajustes constantes que esto implica, poniendo énfasis en el contacto y necesario diálogo entre las culturas antes que en los límites infranqueables entre ellas a los que alude la educación multicultural (Juliano, 1993).

En este trabajo destacamos que la perspectiva intercultural abre la posibilidad de un mutuo enriquecimiento en el encuentro entre culturas diferentes. La habilitación de este diálogo se ve favorecida cuando se respetan tanto la diversidad cultural como la igualdad en la condición cultural. Utilizamos la noción "dinámicas interculturales" para hacer referencia al ejercicio de comprensión de lo igual y lo diverso en espacios socioculturales heterogéneos. En el terreno de la interculturalidad, los procesos de integración se relacionan con la cuestión del reconocimiento de la diversidad y la igualdad como dos dimensiones intrínsecas a la condición humana (Clérico e Ingüi, 2018). Intentamos, de esta manera:

"Evitar confundir la legítima reivindicación de lo específico y original de cada tradición con la idea de que cada comunidad debe encerrarse en su diferencia y afirmarse en su oposición a otras tradiciones, lo cual es contrario a la naturaleza misma del ser humano. Esto remite a la necesidad de pensar en la posibilidad de encuentro entre las culturas más que en su mera cohabitación". (Clérico, 2014:119)

En síntesis, podemos subrayar que la interculturalidad ofrece una perspectiva de apertura al otro, de diálogo y mutuo enriquecimiento y propicia la interacción donde el otro es reco- 
nocido como otro yo, con sus mismas exigencias humanas fundamentales. Se avanza así hacia una superación de los riesgos que conllevan los excesivos particularismos relativistas o su contracara, la homologación unificadora.

\section{El voluntariado universitario como espacio formativo en competencias interculturales}

A lo largo del camino recorrido, advertimos que las dinámicas interculturales emergen como una cualidad esencial de las experiencias de voluntariado y se van tornando en una de las categorías nucleares de nuestra propuesta. Es que "los procesos de voluntariado otorgan a las universidades la oportunidad de formar a sus estudiantes en valores tan importantes como el respeto, la tolerancia o la empatía con el otro" (Gaete Quezada, 2015:242). Es en este aspecto que encontramos el fundamento para afirmar que es posible conectar los procesos de educación intercultural con el voluntariado universitario.

Entre los diversos programas interculturales, una mención especial merece el que apunta al desarrollo de competencias interculturales, ${ }^{10}$ ya que "facilita las relaciones e interacciones entre personas de varios orígenes y culturas, así como dentro de grupos heterogéneos, todos ellos necesitan aprender a vivir juntos en paz" (Universidad Nacional de Colombia y Cátedra UNESCO, 2017:11).

Y tal como las define UNESCO:

"Las competencias interculturales están íntimamente integradas al aprendizaje del saber, hacer y ser. Aprender a saber sobre otros culturales es el primer paso para lograr competencias interculturales, un paso que nunca está completo porque siempre hay otros por encontrar. Aprender a hacer sirve como etapa activa de interacción con otros culturales, a través de tales interacciones la gente práctica el conocimiento ganado y adquiere más, aprendizaje de interacciones con otros en el pasado y diseño de interacciones futuras. Aprender a ser se apoya en una fase reflexiva sobre el ser social de uno al tener un lugar en el mundo global". (p. 13)

En relación con los destinatarios de estas acciones, las soluciones pedagógicas transversalizan la atención a la diversidad al promover "competencias interculturales" en todos los alumnos (Dietz y Mateo Cortés, 2011:102) y no sólo a los grupos minoritarios.

10) La perspectiva intercultural se plasma en diversos modelos educativos, tales como la educación antirracista, la educación holística, la educación intercultural bilingüe, entre otros. El primero parte de la premisa que las sociedades actuales son racistas y el sistema educativo es reproductor de esa ideología. Rechaza el énfasis en la diversidad cultural del multiculturalismo y plantea como objetivos combatir la ideología racista que se transmite subliminalmente desde la educación y educar en la percepción de las estructuras ideológicas e históricas subyacentes en la mirada racista. El segundo modelo (formulado por Banks, 2005) plantea la necesidad de que en la educación se integre el enfoque intercultural y el sociocrítico. Banks desarrolla una serie de principios que apuntan a que se desarrolle una educación holística. En ellos se vislumbra el interés por involucrar activamente a los docentes para lograr el impacto deseado. En ese sentido, fomenta su formación en relación con la cuestión étnica como condición para desarrollar prácticas interculturales y la utilización de distintas técnicas que les permita evaluar teniendo en cuenta la cultura de sus alumnos. El tercer modelo, intercultural bilingüe, se desarrolla particularmente en América Latina con relación a las poblaciones indígenas. Las experiencias son orientadas a atender de modo más adecuado a los diferentes grupos sociales y culturales marginados (Clérico, 2014). 
El análisis de la información recogida en distintas instancias de este proceso (encuestas de expectativas, evaluaciones, informes institucionales, etc.) evidencia que algunos aprendizajes adquiridos durante esta trayectoria pueden claramente vincularse a la educación que propicia el encuentro intercultural.

Así puede reconocerse en las palabras de los protagonistas, que transcribimos a continuación para tratar de ejemplificar cómo se expresan las competencias interculturales de apertura, empatía, diálogo y reflexividad crítica.

En su experiencia como voluntarios, conocen modos de significación de la realidad particulares propios de los grupos con los que trabajan, que muestran posicionamientos personales y colectivos diferentes de los que ellos están habituados.

Es preciso dar a conocer, además, que un alto porcentaje de los estudiantes que se involucran en estos proyectos de voluntariados procede de localidades del interior o de otras provincias, lo cual puede explicar que en estos casos estas diferencias les resulten llamativas.

Respecto de la apertura, los estudiantes expresan la disposición a conocer modos de significación de la realidad distintos del propio como esencial en su formación:

"Mis expectativas son: además que es una muy buena oportunidad para conocer una realidad que no es la mía, tener otras perspectivas de lo que es Santa Fe ciudad, y desde mi futura profesión poder tener las herramientas para pensar en un mejor modo de llevar a cabo el bienestar colectivo". (Encuesta inicial a voluntarios, 2019)

"Me interesa ayudar ya sea en conocimientos o tan solo aportar algo, e interactuar con una realidad que no conozco de cerca". (Encuesta inicial a voluntarios, 2019)

Justamente esta práctica de extensión es concebida como una posibilidad de acercamiento a realidades hasta el momento consideradas como "extrañas" y "lejanas" y que de pronto comienzan a tornarse "familiares" y "accesibles":

"[En este voluntariado] aprendí a conocer otros contextos, otras realidades que están más cerca de lo que parece. y que con la educación y el trabajo y esfuerzo se puede cambiar esas realidades y llegar muy lejos como persona mundo". (Encuesta a voluntarios, evaluación final, 2017)

Algunos estudiantes expresan que tuvieron oportunidad de vincularse con personas cuyas formas de interpretación de la realidad son diferentes de la propia, lo cual les genera una apertura hacia una visión integral:

"Una experiencia significativa en mi desempeño como voluntario de este proyecto fue el poder establecer relaciones con algunos estudiantes en donde comprendí otra forma de ver la realidad, otra cosmovisión del mundo". (Encuesta a voluntarios, evaluación final, 2017)

Resulta interesante que ya antes de empezar el voluntariado, los estudiantes incluyen entre sus expectativas esta ocasión de ampliar su mirada de la realidad:

"[Espero de este voluntariado] crecimiento personal, cambiar el modo de ver el mundo y aprender a enseñar". (Encuesta inicial a voluntarios, 2019) 
La empatía es otra competencia intercultural que apareció mencionada en forma recurrente entre los aprendizajes de los voluntarios y que constituye la capacidad de una persona para mirar desde el punto de vista del otro, es decir, intentar percibir y comprender los sentimientos y deseos de otra persona, lo que juega un papel importante en la resolución de conflictos (Gil-Madrona y Gómez Barreto, 2017). Las siguientes frases dan cuenta que esta competencia en el inicio, como motivación, y al finalizar la práctica, como resultado:

"Las expectativas son buenas, espero crecer como persona, poder tener más empatía y mejorar en todos los sentidos de mi vida. Espero que mi paso por el voluntariado sea significativo al menos para alguno de ellos". (Encuesta inicial a voluntarios, 2019)

"Una experiencia que me resultó significativa en mi desempeño como voluntario en este proyecto fue el aprendizaje de 'ponerse en sus zapatos' y entender su visión del mundo". (Encuesta a voluntarios, evaluación final, 2018)

"Como voluntaria, aprendí sobre la importancia (y la necesidad) de reconocer al otro como un interlocutor válido como condición para afrontar un trabajo conjunto". (Encuesta a voluntarios, evaluación final, 2018)

La posibilidad de aprender estas competencias acontece no solo durante el encuentro entre estudiantes universitarios y secundarios, sino también a través de las interacciones que se generan entre los mismos voluntarios y con los docentes, en las instancias de capacitación:

"De la capacitación [destaco] el intercambio de experiencia entre voluntarios, fue lo que me resultó más interesante, ya que es bueno siempre escuchar experiencias de otros para enriquecernos más en el tema". (Encuesta a voluntarios, evaluación final, 2017)

Esta vivencia de empatía por la cual la percepción propia de que el otro, en tanto persona, es "otro yo", tiene como fundamento originario el hecho de que los seres humanos se reconocen mutuamente en cuanto sujetos simultáneamente diferentes y semejantes (Gaspar et al., 2013:181).

Otra competencia intercultural reconocida como aprendizaje es el diálogo intercultural, que se refiere específicamente a diálogos ocurridos entre distintos grupos culturales. Es un proceso que incluye un intercambio abierto y respetuoso de visiones entre individuos y grupos de diferentes contextos y herencias étnicas, culturales, lingüísticas, sobre la base de la comprensión y el respeto mutuos (Universidad Nacional de Colombia y Cátedra UNESCO, 2017).

En el marco de este proyecto, los estudiantes universitarios aprenden a entrar en diálogo con grupos de distintos contextos sociales destinatarios de las acciones extensionistas, lo cual los prepara para desplegar esta habilidad en posibles contactos futuros con grupos de diferentes culturas. De hecho, en sus informes finales, los directivos expresan que es uno de los principales logros de esta intervención:

"Para la escuela, la experiencia fue muy positiva, pensamos que se constituye en una estrategia para motivar cambios de actitud en los alumnos, mejorar el clima del aula, propiciar intercambios comunicacionales enriquecedores, fortalecer actitudes de auto superación y consolidar mejores modos de relación entre todas las personas involucradas en la tarea escolar". (Informe institucional - EETP N 481) 
"El trabajo del voluntariado es positivamente significativo a nivel pedagógico porque dinamiza y enriquece el trayecto del alumno en la materia, en el grupo y en la escuela, priorizando desde lo humano la atención y el acompañamiento necesario". (Encuesta Directivos - EETP N 481)

Asimismo, los estudiantes identifican el valor del diálogo como uno de los principales aprendizajes de su experiencia:

"Aprendí que quizás el problema más complicado puede resolverse solo con escuchar lo que los chicos tienen para decirnos. Que hay que ser empático y no crear abismos entre el educador y el educado sino crear puentes de comunicación y mutuo crecimiento". (Encuesta a voluntarios, evaluación final, 2018)

De esta manera, un voluntario da a conocer el modo en que el diálogo con los adolescentes pudo ser una herramienta clave para ayudar a mejorar aspectos de su vida:

"Comentaré simplemente la primera que recordé: María en 2013 diciéndome que no leía porque no veía. Lo primero que hice fue dudar hasta que me contó una larga historia de la pérdida de sus lentes casi un año antes. Entre risas, le dije que se haga los lentes porque si no iba a leer igual; a las tutorías siguientes me cuenta en la puerta del aula que había ido al hospital para que le hagan sus nuevos lentes. Más allá de cómo continúa la historia, para mí es bisagra en las tutorías porque muestran que no solo es apoyo escolar, muestra que con un diálogo alguien puede empezar a tomar decisiones para su vida". (Encuesta a voluntarios, evaluación final, 2018)

En este sentido, entre las expectativas que expresan al inicio de su práctica, denotan una valoración del imprevisto que puede suscitarse en el encuentro humano, más allá del rédito inmediato de su accionar en la escuela destino:

"Quiero dejar de lado las expectativas y sorprenderme en el transcurso. Quiero volver de la escuela sabiendo que pude haber mejorado el día de alguien, quiero poder lograr un vínculo con los chicos, estar con ellos para lo que necesiten ya sea risas, aliento o silencio". (Encuesta inicial a voluntarios, 2019)

En el momento de dar inicio a esta práctica, muestran el valor que tuvieron sus experiencias anteriores, que marcaron el gran interés por este tipo de prácticas:

"Quiero ser voluntario de este proyecto porque me gusta dar un apoyo escolar, o simplemente estar para la persona que necesita de un consejo o simplemente de que lo escuchen. Más allá de los voluntariados que hice y me dejaron marcas de por vida y cada una con enseñanzas diferentes". (Encuesta inicial a voluntarios, 2019)

Es claro que en los encuentros que se desarrollan semanalmente en la práctica de voluntariado se propician procesos de horizontalidad y comunicación dialógica con los otros actores que participan de esta experiencia. Y respecto de la competencia de reflexividad crítica, Gaspar et al. (2013) destacan que "la acción voluntaria conmueve y suscita la reflexión, moviliza a la persona a preguntarse sobre lo que realiza y a posicionarse a partir de lo que comprende" (p. 178). 
Ciertos estudiantes señalan este aspecto como principal motivación inicial para participar en el voluntariado:

"[Me anoté en este voluntariado] porque quería adquirir experiencia en el encuentro con el otro, brindar mis conocimientos y herramientas para ayudar a los jóvenes en proceso de escolarización, y no solo desde el punto de vista académico sino brindar una contención mayor, y a su vez aprender a solucionar situaciones y así crecer personalmente y en mi futuro profesorado". (Encuesta inicial a voluntarios, 2018)

También, durante el recorrido surgen nuevos aprendizajes que movilizan a los sujetos implicados, tal como lo ponen en evidencia Gaspar et al. (2013):

"Trabajando voluntariamente, los sujetos se sorprenden en el encuentro con el otro y, siguiendo la invitación que recogen de esa experiencia, se empeñan en construir relaciones personales en las cuales se evidencia su conmoción delante del otro, su consideración por ellos y su disposición tanto para aprender cuanto para enseñar. Delante del otro, el 'yo' no se queda quieto". (p. 180)

La reflexión crítica se reconoce en relación con la posibilidad de participar en la construcción de alternativas a un mundo "materialista" y "egoísta", como ellos lo expresan:

"El voluntariado me permitirá conocer más a fondo la comunidad en la que vivo - quiénes la conforman, cuáles son sus necesidades, etc.-. A su vez me ayudará a reforzar la pertenencia a una comunidad es muy importante hoy en día, cuando la vida acelerada y materialista produce tanta alienación y egoísmo". (Encuesta inicial a voluntarios, 2019)

Algunos alumnos profundizan en cuanto a considerar el voluntariado como una experiencia de formación integral, que trasciende (y complementa) lo académico:

"Principalmente [me anoté como voluntario] para complementar mi formación profesional ya que considero que hay carencias en mi plan de estudios. Lo pienso como un espacio de aprendizaje muy importante a nivel personal, ya que veo necesario acercarme a las realidades con las que me encontraré a futuro $y$, mientras pueda dejar un impacto positivo, aunque sea en un solo alumno, sé que valdrá la pena. Quiero participar en la lucha contra la estigmatización de quienes están en situación de vulnerabilidad desde el lugar que ocupo, ya sea como parte del sistema educativo universitario, como la persona que soy hoy". (Encuesta inicial a voluntarios, 2019)

Es así como esta práctica de extensión les permite aproximarse a las personas desde una actitud abierta, crítica y reflexiva, lo cual les ayuda a superar los sesgos estigmatizantes con que se califica a las poblaciones que habitan los barrios periféricos de la ciudad.

La posibilidad de interactuar con diferentes actores sociales que ofrecen las acciones de extensión, al decir de Menéndez (2013), interpela a la propia institución universitaria, sus prácticas e intervenciones, ya que los estudiantes se encuentran con problemáticas concretas en un contexto complejo y particular que demanda articulación de conocimientos y saberes de distintas disciplinas. Compartir la mirada de un problema con compañeros de diferentes carreras ejercita modalidades comunicativas y conceptuales, habilita la construcción de consensos y favorece el diálogo interdisciplinario. Muchas veces también aparecen 
contradicciones, perspectivas opuestas entre lo escolar y lo universitario, que exigen de los voluntarios la tolerancia y la suspensión del juicio, indispensable para ciertos momentos del accionar en común y del reconocimiento del otro en su saber, su ser, su hacer.

De esta manera, la experiencia propone a los voluntarios anticipar cierto esfuerzo interdisciplinario ya durante su carrera académica, lo cual seguramente redundará en beneficio de su formación específica y, por supuesto, de su desempeño profesional futuro.

Es preciso considerar además la posibilidad de establecer los lazos sociales y de la flexibilidad que requiere la interacción con el otro para construir un hacer situado en un determinado territorio, que antes era percibido como ajeno y que poco a poco puede reconocerse como un espacio factible de desarrollo y de trabajo.

En síntesis, la extensión tiene, en potencia, una dimensión educativa de importancia excepcional. En el proceso de solución de los problemas sociales, los universitarios pondrán en juego habilidades, conocimientos, actitudes y valores. Esta intervención logrará que la formación de nuestros alumnos sea más sólida y profunda (Serna Alcántara, 2007). Asimismo, fomenta una actitud clave para la formación académica: la de búsqueda constante, tal como lo destacan Tommasino y Stevenazzi (2016):

"La extensión puede transformarse, acercando a los estudiantes al contacto con las diferentes realidades sociales y a la conexión con lo que van aprendiendo, en relación con la sociedad y sus formas de entenderla. Este acercamiento necesariamente desafiará a estudiantes y docentes, colocándolos en el lugar de la incerteza y la búsqueda, actitudes fundamentales en cualquier proceso de formación. Esas realidades sociales se convierten en el principal instrumento didáctico para orientar la formación". (p. 123)

De tal forma, podemos concluir este apartado subrayando que la interculturalidad "debe ser un núcleo de la comprensión de las prácticas y la elaboración de políticas", al decir de García Canclini (2004:73), quien subraya la necesidad de una reorientación del trabajo en las ciencias sociales en la acción social y política para aceptar los nuevos problemas de la contemporaneidad. Ante la provocación de su pregunta: "¿qué significa construir —o reconstruir-identidades en las condiciones de un tiempo de globalización?", y vinculándola a la cuestión aquí presentada, nos podemos interrogar: ¿qué significa educar en este tiempo de globalización? La hipótesis delineada en este artículo permite anticipar una posible respuesta: ofrecer a los estudiantes la posibilidad de aprender competencias que les enseñen a entrar en relación con el otro de modo abierto y empático, dialógico, crítico y reflexivo. El voluntariado universitario brinda una excelente oportunidad para ello.

\section{Aportes del voluntariado para la integralidad de la formación universitaria}

Cuando hacemos referencia a la integralidad, destacamos que participar en propuestas de extensión "hace diferencia" en cada uno de quienes formamos parte de ellas y contribuye a ligar diferentes saberes y conocimientos en función de una situación concreta en la que intentamos intervenir (Bonelli et al., 2018). Nos interesa destacar la búsqueda de dicha integralidad en la formación y en la transmisión, que se evidencia cuando pensamos el hecho extensionista como un encuentro humano, donde cada uno de nosotros aparece como su- 
jeto de aprendizajes y vivencias, con un cúmulo de significaciones que ponemos en juego a cada momento. Tal como lo resaltamos anteriormente:

"El trabajo colectivo entre voluntarios, coordinadores, profesores, docentes y alumnos de las instituciones involucradas suscita un aprendizaje en terreno de lo que significa 'hacer con', hacer con sus compañeros voluntarios, con los docentes y los preceptores a cargo de los adolescentes, con sus capacitadores y sobre todo, con los propios adolescentes destinatarios de esta acción. Aprender a hacer con otros amplía el horizonte de los estudiantes universitarios, ofreciéndoles una ocasión de aprendizaje en terreno (...). En estos aprendizajes, el encuentro con el otro permite la puesta en juego de dinámicas interculturales que enriquecen y amplían el horizonte de sentido del estudiante universitario y del alumno secundario, pero también del docente secundario y de nosotros, los docentes universitarios, propiciando los múltiples encuentros mutuos, encuentros que se configuran en una posibilidad de aportar a la integralidad de la experiencia formativa universitaria". (Bonelli et al.:11)

Entendemos que es la progresiva participación en estas actividades de carácter comunitario la que favorecerá la construcción de aprendizajes (Rafaghelli, 2016). En efecto, a través de sus relatos, los estudiantes nos confirman que este tipo de experiencias educativas en extensión representa una posibilidad de favorecer el entusiasmo por enseñar y aprender. En esa misma línea, es posible sostener, con Tommasino y Stevenazzi (2016), que "la extensión contribuye a los procesos de formación en la medida que habilita enseñanzas y aprendizajes en múltiples direcciones" (p. 123).

Cuando la articulación del voluntariado con la formación es correctamente gestionada dentro de las universidades, esta adquiere un rol central:

"Se convierte en los campus universitarios en un instrumento educativo y formativo que complementa la formación que los estudiantes reciben en las aulas y que no estaba contemplada de esta manera hasta este momento. Así, los años de estudios universitarios se convierten también para los jóvenes en años de aprendizaje social, durante los cuales se fomenta la actuación del universitario en la transformación de la sociedad". (Arias, 2008:24, citado por Gaete Quezada, 2015)

Al respecto, es necesario poner de relieve, tal como lo hacen Tommasino et al. (2010), que en los últimos años la extensión ha transitado un proceso de reconceptualización que enfatizó sus aportes pedagógicos en virtud de las diversas experiencias educativas en comunidad. Describe este proceso como el pasaje de la extensión a las prácticas universitarias integrales y retoma con la idea de integralidad en las prácticas educativas, de una larga tradición en la Universidad.

Los autores advierten que esta noción implica una articulación particular entre los procesos de aprendizaje y enseñanza, de investigación y extensión, que incluye un abordaje interdisciplinario y multiprofesional, supone una relación dialógica y crítica entre actores vinculados, y pone en juego una relación dialéctica de saberes académicos y populares. También destaca que en las prácticas integrales donde se articulan las funciones el rol de la extensión puede propiciar la reconfiguración, integración y renovación de todos los componentes del acto educativo. Las experiencias educativas en terreno posibilitan procesos que 
interpelan los conocimientos adquiridos en las aulas y su mejor internalización; al vincular a los estudiantes con problemáticas sociales de su tiempo y ponerlos a trabajar junto a las comunidades que las viven, promueven procesos de transformación subjetiva y reflexión ético-crítica favorecedoras de la asunción de posicionamientos personales y colectivos críticos, responsables y solidarios. Con relación a ello, Tomasino subraya que "la articulación de funciones que supone la integralidad genera relaciones diferentes de las que pueden darse en un aula universitaria y en función de ello toda esa trama de relaciones se altera" (Tommasino y Stevenazzi, 2016:123). Puede reconocerse, con Gaete Quezada (2015), que:

"Las acciones de voluntariado universitario se transforman en un espacio de aprendizaje para ellos, lo que les permite complementar su formación teórica proporcionada por la universidad, con la puesta en práctica de sus habilidades al servicio de la comunidad para intentar producir un cambio social, paralelamente con un aumento de su propia conciencia ciudadana y social respecto de su entorno". (p. 245)

Es así como el voluntariado universitario constituye un ámbito interesante para fortalecer los procesos integrales de formación universitaria, lo cual repercute positivamente en la comunidad académica y, al mismo tiempo, en el conjunto de la sociedad.

\section{Reflexiones finales}

En este artículo intentamos transmitir el modo en que se viene implementando en Santa Fe una experiencia de intervención en terreno ligada a la inclusión socioeducativa y cultural. En las páginas precedentes se ha trazado, primero, una síntesis de la historia y los rasgos que fue adquiriendo esta práctica de extensión a lo largo de esta década de trabajo; y luego se han revelado desde las voces de algunos de sus actores las implicancias del voluntariado para la educación intercultural, con los desafíos que esto supone para avanzar en una formación universitaria integral.

El trabajo colectivo entre voluntarios, coordinadores, docentes y alumnos de las instituciones involucradas, universidades, ACDI y las escuelas participantes, suscita múltiples aprendizajes experienciales. La reciente incorporación de nuevos actores sociales, AAPS y Asociación Civil "Espacios Ekekos", permite profundizar en los objetivos del segundo eje de trabajo: la educación artística, y ofrece valiosas contribuciones en el camino de potenciar el alcance intercultural de esta propuesta de intervención.

A partir de este recorrido podemos concluir que la práctica de voluntariado, en especial aquella que se desenvuelve en contextos culturalmente heterogéneos, presenta un importante potencial a los fines de la educación intercultural. Así, los aprendizajes de los cuales dan cuenta los estudiantes universitarios que se incorporan como voluntarios constituyen un hito inescrutable de lo que significa la formación en competencias interculturales. Aprendizajes ligados a tener una posición de apertura en el encuentro con el otro, una empatía hacia sus necesidades y un acortamiento de las distancias entre los sujetos desde una actitud crítica y reflexiva.

Estudiantes procedentes de distintas localidades de la provincia de Santa Fe o de otras provincias, o que residen en la ciudad, encuentran en las prácticas de voluntariado la posibilidad de acercarse a realidades consideradas "lejanas", "extrañas", y hasta "hostiles", en 
virtud de la fuerte connotación negativa con que las representaciones sociales que circulan en los medios de comunicación asignan a la población de los barrios urbano-marginales. De esa forma, este acercamiento quiebra con representaciones y prácticas estigmatizantes. La multiplicidad de relaciones puestas en juego en estos encuentros con los adolescentes y los docentes de las escuelas donde desarrollan su tarea como voluntarios crea climas propicios para mirar y tratar al otro de un modo diferente: el extraño pasa a ser familiar; el lejano, cercano; el enemigo, amigo.

Se pone así en evidencia que las acciones de extensión ofrecen escenarios de interculturalidad óptimos para propiciar el aprendizaje de estas competencias. La oportunidad que brinda el voluntariado para potenciar los aprendizajes experienciales de competencias interculturales se torna claramente en un valor agregado importante, lo cual alienta a continuar desarrollando este tipo de instancias al interior de nuestras universidades.

La necesidad de compartir nuestra experiencia surge respecto de la posibilidad de realizar una resignificación de los alcances de las acciones de extensión universitaria, tomando como núcleo para su comprensión la contribución que ofrece a la educación intercultural de los actores involucrados. Tal como hemos podido identificar en los relatos de los estudiantes, la práctica de voluntariado es una contribución crucial para la integralidad de la formación universitaria.

\section{Referencias bibliográficas}

ACDI (s/f). Padrinos solidarios. Recuperado de: http://acdi.org.ar/padrinos-solidarios/

Barth, F. (1995). Les groupes etniques et leurs frontiers. En Poutignat, P. y Streiff-Fenart, J. Theories de l'ethnicité (pp. 203-209). Paris: PUF. Col. Le sociologue.

Bonelli, M.; Clérico, G.; Prat, T.; Rey, S. y Fontán, N. (2018). Experiencia intercultural en extensión: un aporte a la integralidad de la formación universitaria. En Herrero, D.E. y Varela, J.R. Conclusiones de las VI Jornadas de Extensión de Mercosur. I Coloquio Regional de la Reforma Universitaria. 1ra ed. Tandil: Universidad Nacional del Centro de la Provincia de Buenos Aires.

Clérico, G. (2014). La diversidad cultural y la igualdad humana en la educación intercultural. Estudio crítico sobre la base de la experiencia de niños migrantes en una escuela de la ciudad de Córdoba. Tesis Doctoral en Educación no publicada. Santa Fe: Universidad Católica de Santa Fe.

Clérico, G.M.; Ingüi, P. (2018). Interculturalidad e internacionalización curricular en la enseñanza de Psicología: transformar realidades e interrogar la disciplina. En Anales III Congreso Internacional de Psicología. VI de Congreso Nacional de Psicología, Ciencia y Profesión “Desafíos para la Construcción de una Psicología Regional”. Córdoba: Universidad Nacional de Córdoba.

Clérico, G.M.; Ingüi, P.; Gaspar, Y.E.; Assis, R.M. (2017). Aprendiendo a reconocer las dinámicas interculturales en una experiencia de internacionalización curricular entre universidades de Argentina y Brasil. En Anales VIII Encuentro Nacional y V Latinoamericano "La universidad como objeto de investigación". Santa Fe: UNL. Cuche, D. (2002). La noción de cultura en las ciencias sociales. Buenos Aires: Nueva Visión.

Dietz, G. (2012). Multiculturalismo, interculturalidad y diversidad en educación: una aproximación antropológica. México: Fondo de Cultura Económica.

Dietz, G. y Mateos Cortés, L. (2011). Interculturalidad y Educación Intercultural en México. Un análisis de los discursos nacionales e internacionales en su impacto en los modelos educativos mexicanos. México: Secretaría de Educación Pública. Coordinación General de Educación Intercultural y Bilingüe. 
Gaete Quezada, R. (2015). El voluntariado Universitario como ámbito de aprendizaje servicio y emprendimiento social. Un estudio de caso. Última década, 23(43). Santiago, Chile. Recuperado de: http://dx.doi. org/10.4067/S0718-22362015000200009

García Canclini, N. (2004). Diferentes, desiguales y desconectados. Mapas de la interculturalidad. Madrid: Gedisa.

Gaspar, Y.; Rodriguez, N. Alvez Macondes, L.; Bittar Bueno, C. (Orgs.) (2013). Ser voluntário, ser realizado. Investigacao fenomenologica numa institucao espirita. São Paulo: Unifran.

Geertz, C. (2000). La interpretación de las culturas. Barcelona: Gedisa.

Gil-Madrona, P. y Gómez Barreto, I. (2017). La competencia intercultural en la formación de los maestros como condicionante de la mejora de la interacción docente en las aulas. Actas de las V Jornadas de Didáctica del Español como Lengua Extranjera del Instituto Cervantes de Budapest. Madrid: Centro Virtual Cervantes. Recuperado de: https://cvc.cervantes.es/ensenanza/biblioteca_ele/publicaciones_centros/PDF/budapest_2017/03_gilmadrona.pdf

Juliano, D. (1993). Educación intercultural. Escuelas y minorías étnicas. Madrid: Eudema Antropología.

Leite, R.V. (2016). Pesquisa fenomenológica de um encontro intercultural: a experiência de crianças da comunidade tradicional de Morro Vermelho. Curitiba: Appris.

Mato, D. (Coord.) (2018). Educación superior, diversidad cultural e interculturalidad en América Latina. Conferencia Regional de Educación Superior (CRES) 2018. Caracas, UNESCO-IESALC; y Córdoba, Universidad Nacional de Córdoba.

Malgesini, G.; Giménez, C. (2000). Guía de conceptos sobre migraciones, racismo e interculturalidad. Madrid: Los libros de la Catarata.

Menéndez, G. (2013). Institucionalización de la Extensión. Conceptualización y dimensiones de la Extensión. En Menéndez, G. et al. Integración, docencia y extensión. Otra forma de aprender y de enseñar. Santa Fe: Ediciones UNL. Recuperado de https://bibliotecavirtual.unl.edu.ar:8443/colecciones/bitstream/handle/123456789/7967/extension_interior_pag_83_91.pdf

Muñoz Sedano, A. (1999). La educación intercultural. Enfoques y Modelos. En Checa, F. y Soriano, E. (Eds.). Inmigrantes entre nosotros. Barcelona: Icaria.

Rafaghelli, M. (2016). Las prácticas de extensión en Educación Experiencial como oportunidad para integrar docencia y extensión. +E: Revista de Extensión Universitaria, 6(6), 8-15, Santa Fe, Argentina: Ediciones UNL. Sales Ciges, A. y García López, R. (1997). Programas de Educación Intercultural. Bilbao: Desclée De Brouwer. Serna Alcántara, G.A. (2007). Misión social y modelos de extensión universitaria: del entusiasmo al desdén. Revista Iberoamericana de Educación, 43(3). Organización de Estados Iberoamericanos para la Educación, la Ciencia y la Cultura (OEI). Recuperado de: https://dialnet.unirioja.es/servlet/articulo?codigo=2316425

Schmelkes, S. (2003). Educación intercultural. Reflexiones a la luz de experiencias recientes. Sinéctica, (23, agosto-enero), 26-34. México: Instituto Tecnológico y de Estudios Superiores de Occidente. Recuperado de: https://sinectica.iteso.mx/index.php/SINECTICA/article/view/296/289

Soler Castillo, S. (2918). Racismo y educación. Una revisión crítica. Educação em Revistal Belo Horizonte, (34), e192221. Recuperado de: http://www.scielo.br/pdf/edur/v34/1982-6621-edur-34-e192221.pdf

Tommasino, H. et al. (2010). De la extensión a las prácticas integrales. La reforma universitaria. La extensión en la renovación de la enseñanza. Espacios de formación integral. No 10 (pp. 25-31). Montevideo: Rectorado. Universidad de la República. Recuperado de: http://www.extension.udelar.edu.uy/wp-content/uploads/2016/12/08_ Hacia-la-reforma-universitaria_-la-extensio\%CC\%81n-en-la-renovacio\%CC\%81n-de-la-ensen \% CC\%83anza.pdf 
Tommasino, H. y Stevenazzi, F. (2016). Reflexiones en torno a las prácticas integrales en la Universidad de la República. +E: Revista de Extensión Universitaria, 6(6), 120-129. Santa Fe, Argentina: Ediciones UNL. Recuperado de: https://bibliotecavirtual.unl.edu.ar/publicaciones/index.php/Extension/article/view/6320

Universidad Nacional de Colombia y Cátedra UNESCO (2017). Diálogo intercultural, Competencias Interculturales: marco conceptual y operativo. Recuperado de: https://www.dialogointercultural.co/pdf/Competencias_interculturales.pdf

Universidad Nacional del Litoral (s/f). Régimen de Voluntariado Universitario. Recuperado de: https://www.unl. edu.ar/extension/wp-content/uploads/sites/9/2018/11/1243_regimen_de_voluntariado.pdf 